\title{
Prevalence of Methicillin Resistant Staphylococcus aureus (MRSA) and Multidrug Resistance among them in a Tertiary Care Rural Hospital
}

\author{
Prachi Arvind Anchawale* \\ Department of Microbiology, Dr. Vitthalrao Vikhe Patil Foundation's Medical College and \\ Hospital, Ahmednagar, Maharashtra, India \\ *Corresponding author
}

\begin{abstract}
A B S T R A C T
Keywords

Methicillin

Resistant

Staphylococcus

aureus (MRSA)

Article Info

Accepted:

07 October 2020

Available Online:

10 November 2020

Infections caused by Staphylococcus aureus have a poorer prognosis when the infecting strain is Methicillin-resistant Staphylococcus aureus (MRSA) additionally MRSA strains are important for their resistance to many other commonly used antibiotics. The objectives of this study were to estimate the prevalence of MRSA strains in clinical specimens and to investigate their sensitivity pattern. A total of 480 various samples received in the department of Microbiology at MIMER Medical college and hospital, Talegaon were included in the study. The MRSA strains were identified by using Oxacillin resistance screening agar and Cefoxitin $(30 \mu \mathrm{g})$ disc diffusion. Their sensitivity pattern was investigated using disc diffusion and minimum inhibitory concentration (MIC) by $\mathrm{E}$ test. Prevalence of MRSA in our study is $19.37 \%$. There is significant difference between rates of isolation of MRSA from various clinical specimens. All MRSA strains tested against vancomycin and linezolid showed $100 \%$ sensitivity. Highest resistance among MRSA was found for amoxicillin-clavulanic acid (74.19\%). There is a need to reduce the burden of infections caused MRSA. Continuous efforts should be made to prevent the spread and the emergence of resistance by early detection and using the proper infection control measures.
\end{abstract}

\section{Introduction}

In the genus Staphylococcus, Staphylococcus aureus ( $S$. aureus) is the most important pathogenic organism and it is also one of the most common versatile nosocomial pathogen. ${ }^{1}$ It is responsible for most common causes of blood stream infection (BSI), skin and wound infections, osteomyelitis, endocarditis, and nosocomial infections, especially pneumonia, surgical site infections, and continues to be a major cause of community-acquired infections., ${ }^{2,3}$
It has overcome most of the therapeutic agents that have been developed over the years. The important notable example was the methicillin resistant Staphylococcus aureus (MRSA) which was reported just one year after the launch of methicillin. ${ }^{1}$ S. aureus strains expressing the mecA determinant are termed as MRSA. ${ }^{4}$ The worst feature of MRSA is simultaneous drug resistance to many antibiotics and chronic carrier stage among the healthcare workers ${ }^{1}$. Accurate and prompt detection of Methicillin Resistance Staphylococcus aureus (MRSA) in patient's 
sample is of utmost importance in managing these infections and preventing their spread.

Keeping in mind the havoc, MRSA can create, we decided to estimate the prevalence of MRSA in our hospital; and susceptibility pattern of MRSA towards various antibiotics.

\section{Materials and Methods}

Study was conducted in the department of microbiology at MIMER Medical college and hospital, Talegaon. It was a cross-sectional type of study. Study period was from January 2014 to June 2015. All samples received in the microbiology laboratory from various clinical departments of the hospital were processed for isolation of $\mathrm{S}$. aureus and detection of MRSA. Total 480 samples of S. aureus were obtained during study period. Samples included were blood, fluid, stool, pus, sputum, throat swab, urine, miscellaneous samples (it includes, post operative wound swab, high vaginal swab, urinary catheter tips, endotracheal tubes and umbilical catheter tips etc.) Processing of $\mathrm{S}$. aureus isolates were done by using standard methods.

MRSA were detected by using, two methods

Oxacillin resistance screening agar and Cefoxitin disc diffusion.

Antibiotic susceptibility testing was done by Kirby Bauer's disc diffusion method. For determining vancomycin susceptibility, feasible, cost-effective and more reliable $\mathrm{E}$ test method was used.

\section{Results and Discussion}

Detection of mecA gene or its product, penicillin binding protein (PBP2a), is considered the gold standard for MRSA confirmation. ${ }^{5}$ As suggested by CLSI guidelines; cefoxitin-based methods predict the presence of mecA-mediated resistance; their use is preferred to tests using oxacillin because they are better predictors of the presence of mecA than are oxacillin-based methods, including the oxacillin salt-agar screening plate. ${ }^{6}$ Therefore, as depicted in table 1, we have considered prevalence of MRSA based on cefoxitin disc diffusion method i.e. $19.37 \%$.

The findings of isolation of MRSA from various clinical specimens are depicted in table 2. In our study, we found significant difference between rate of isolation of MRSA from various clinical specimens ( $p$ value $<0.05)$. Highest rate of isolation of MRSA from miscellaneous group of samples, i.e. $29.71 \%$. Second highest isolation of MRSA was obtained from blood i.e. $24.73 \%$ followed by pus $(17.77 \%)$.

As shown in graph no. I, we observed significant difference $(\mathrm{p}<0.05)$ in rate of isolation of MRSA from different clinical specialties also i.e. most of the MRSA isolates belonged to surgical units [includes surgery $(46.57 \%)$, orthopedics (33.33\%), obstetrics \& gynecology (21.87\%), ENT (4\%)] rather than medical units [includes ICU (31.37\%) NICU $(28.57 \%)$, medicine $(14.70 \%)]$.

Table 3 depicts antibiotic sensitivity pattern of all MRSA isolates. It showed that all MRSA were sensitive to linezolid followed by amikacin [sensitive (45.16\%) and moderately sensitive (7.52\%)] while highest resistance was found for amoxicillinclavulanic acid (74.19\%) followed by azithromycin and clarithromycin i.e $68.81 \%$ to be followed by cotrimazole and ciprofloxacin i.e. $66.66 \%$. One of the important finding in our study is that, for every antimicrobial agent, percentage of sensitivity was considerably high among total $S$. aureus strains than the percentage of 
sensitivity of MRSA strain. e.g. amoxacilinclavulanic acid sensitive S.aureus were $51.25 \%$ while sensitive MRSA were $25.80 \%$ and for cotrimoxazole, sensitive S.aureus strains were $41.66 \%$ while sensitive MRSA strains were $21.50 \%$. In our study, all the MRSA strains which were tested against vancomycin susceptibility by $\mathrm{E}$ test showed 100\% sensitivity. But out of total 93 MRSA cases $37(39.78 \%)$ were having MIC 1 $\mathrm{mcg} / \mathrm{ml}$ and $32(34.40 \%)$ MRSA strain was sensitive within the range $2 \mathrm{mcg} / \mathrm{ml}$. This suggests that though all these MRSA strains were within the susceptibility range.

Table.1 Prevalence of MRSA

\begin{tabular}{|l|c|c|}
\hline Method & No. of MRSA isolates & Percentage \\
\hline Oxacillin screening agar method & 92 & $19.16 \%$ \\
\hline Cefoxitin disc diffusion & 93 & $19.37 \%$ \\
\hline
\end{tabular}

Table.2 Sample wise distribution (MRSA isolates)

\begin{tabular}{|l|c|c|c|}
\hline \multicolumn{1}{|c|}{ Type of Sample } & $\begin{array}{c}\text { No. of S. aureus isolates } \\
\text { n1= 480 }\end{array}$ & $\begin{array}{c}\text { MRSA isolates } \\
\mathbf{n 2 = 9 3}\end{array}$ & $\begin{array}{c}\text { MRSA } \\
\text { Percentage }\end{array}$ \\
\hline Miscellaneous & 138 & 41 & $(29.71 \%)$ \\
\hline Pus & 135 & 24 & $(17.77 \%)$ \\
\hline Blood & 93 & 23 & $(24.73 \%)$ \\
\hline Urine & 70 & 4 & $(5.71 \%)$ \\
\hline Sputum/ Throat & 32 & 1 & $(3.12 \%)$ \\
swab & & & \\
\hline Fluid & 6 & 0 & 0 \\
\hline Stool & 6 & 0 & 0 \\
\hline
\end{tabular}

Chi-square value $=28.02 p$ value $=0.00009314$ i.e. $p<0.05$

There is significant difference between rate of isolation of MRSA from various clinical specimens $(p$ value $<0.05)$. $\mathrm{n} 1=$ Total no. of S.aureus isolated from various clinical samples $=480$

$\mathrm{n} 2=$ Total no. of MRSA isolated from various clinical samples $=93$

Percentage of MRSA $=$ No. of MRSA isolated from one type of clinical samples $\times 100$

No. S. aureus isolated from the same type of clinical samples

Table.3 Antibiotic sensitivity pattern of all MRSA isolates

\begin{tabular}{|c|c|c|c|}
\hline Antibiotic & No. of Sensitive isolates & $\begin{array}{c}\text { No. of Moderately } \\
\text { Sensitive isolates }\end{array}$ & $\begin{array}{c}\text { No. of Resistant } \\
\text { isolates }\end{array}$ \\
\hline Vancomycin & $93(100 \%)$ & 0 & 0 \\
\hline Linezolid & $93(100 \%)$ & 0 & 0 \\
\hline Amoxicillin -Clavulanic acid & $24(25.80 \%)$ & 0 & $69(74.19 \%)$ \\
\hline Co-trimoxazole & $20(21.50 \%)$ & $11(11.82 \%)$ & $62(66.66 \%)$ \\
\hline Clindamycin & $32(34.40 \%)$ & $12(12.90 \%)$ & $49(52.68 \%)$ \\
\hline Amikacin & $42(45.16 \%)$ & $7(7.52 \%)$ & $44(47.31 \%)$ \\
\hline Azithromycin & $23(24.73 \%)$ & $6(6.45 \%)$ & $64(68.81 \%)$ \\
\hline Ciprofloxacin & $20(21.50 \%)$ & $11(11.82 \%)$ & $62(66.66 \%)$ \\
\hline Clarithromycin & $25(26.88 \%)$ & $4(4.30 \%)$ & $64(68.81 \%)$ \\
\hline
\end{tabular}

[n= No. of Methicillin resistant S. aureus (MRSA) isolates $=93$ ] 
Graph.1 Clinical Specialty wise distribution of MRSA isolates

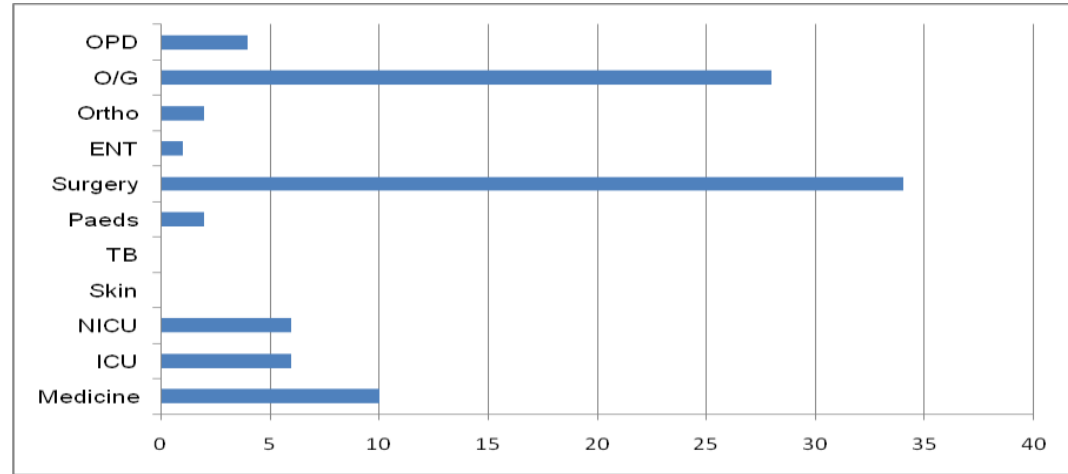

Chi-square value $=65.19 p$ value $=0.000$ i.e. $p<0.05$

Isolation of MRSA sample was significantly different among different clinical specialties. $(p<0.05)$

\section{Image.1 Oxacillin resistance screening agar}

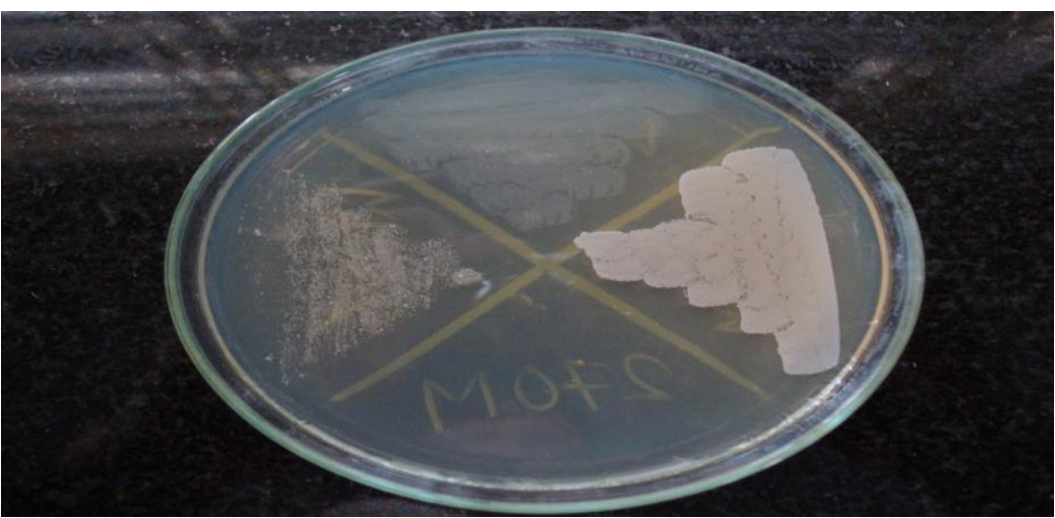

- MRSA strains have grown in $12^{0}$ clock and $3^{0}$ clock and $9^{0}$ clock position quadrants

- S.aureus strain at $6^{0}$ clock is MSSA strain

Image. 2 Cefoxitin disc diffusion testing for MRSA

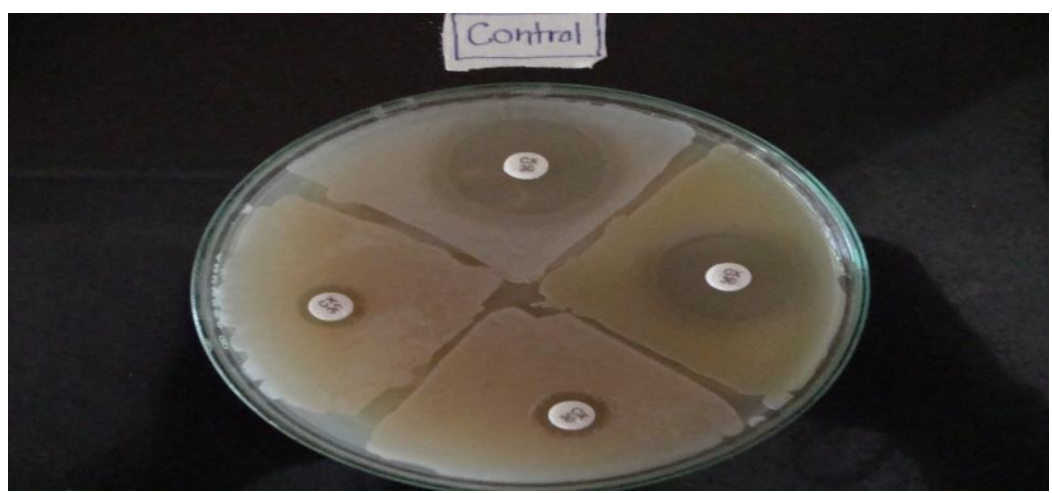

- Strain at $12^{0}$ clock position is a control strain

- S. aureus strain at $3^{0}$ clock position is an MSSA strain

- While strains at $6^{0}$ clock and $9^{0}$ clock position are MRSA strain 
At the time of the introduction of penicillin in the early 1940s, S. aureus was uniformly susceptible to this drug. However, widespread resistance to penicillin developed during the 1950s, followed in the 1970s by increasing resistance to the new semisynthetic penicillinase-resistant antimicrobial agents (i.e., methicillin, oxacillin, nafcillin, dicloxacillin). By $1990 \mathrm{~s}$, resistance to the penicillinase-resistant penicillins spread throughout the world, compromising the use of these drugs for empiric therapy for staphylococcal infections. This has led to increased reliance on vancomycin for treatment of documented methicillin-resistant $S$. aureus (MRSA) infections, as well as for empiric therapy of infections in populations where the prevalence of MRSA is high. ${ }^{7}$

Detection of mecA gene or its product, penicillin binding protein (PBP2a), is considered the gold standard for MRSA confirmation. ${ }^{8}$ A study conducted by $\mathrm{KB}$ Anand et al provides an evidence that cefoxitin can be used as an accurate surrogate marker in routine susceptibility testing at $37^{\circ} \mathrm{C}$ for 18 to 24 hours. In addition, the results have shown $100 \%$ sensitivity and specificity as compared to mecA gene detection by PCR. Hence, it can be used as an alternative to the technically demanding PCR for the detection of MRSA. ${ }^{9}$ Therefore, in our study we considered rate of MRSA prevalence based upon 'Cefoxitin disc diffusion method'. We reported 19.37\% MRSA prevalence in our hospital.

In India, a study was conducted by Indian Network for Surveillance of Antimicrobial Resistance (INSAR) group. This study was conducted in 15 Indian tertiary care centers during a two years period to determine the prevalence of MRSA and susceptibility pattern of $S$. aureus isolates in India. ${ }^{10}$ According to this study, prevalence of MRSA was $42 \%$ in 2008 and in 2009 it is $40 \%$.
In our study, we found significant difference between rate of isolation of MRSA from various clinical specimens ( $p$ value <0.05) Second highest isolation of MRSA was obtained from blood i.e. $24.73 \%$ followed by pus $(17.77 \%)$.

In a study conducted by INSAR (Indian Network for Surveillance of Antimicrobial Resistance), observed highest rate of isolation of MRSA in 2008 and in 2009 was from 'other specimens' (46\%) and blood(48\%) respectively which is similar to our findings is similar to our findings. ${ }^{10}$ While In a study conducted by Sharma P. et al the highest rate of isolation of MRSA was from pus samples (76.92\%) followed by blood $(16.66 \%)$ and sputum $(3.8 \%) .^{11}$ Observations of above mentioned studies are somewhat different from our study.

We found significant difference $(p<0.05)$ in rate of isolation of MRSA from different clinical specialties. In a study carried by $\mathrm{S}$ Srinivasan et al who observed that surgical units (postoperative wound infections) accounted for $80 \%$ of MRSA isolates when compared to $20 \%$ in medical units. ${ }^{12}$ Results observed in the above mentioned studies are in concordance with the findings of our study i.e. most of the MRSA isolates belonged to surgical units [includes surgery (46.57\%), orthopedics $(33.33 \%)$, obstetrics \& gynecology (21.87\%), ENT(4\%)] rather than medical units.

Another study conducted by JB Sarma et al., showed, $100 \%$ of MRSA isolates were resistant to ciprofloxacin; $85 \%$ to clindamycin and $96 \%$ were resistant to co-trimoxazole and amikacin. In contrast, $100 \%$ of MSSA isolates were sensitive to these antimicrobials. ${ }^{13} \mathrm{On}$ the contrary, in our study we found $66.66 \%$ MRSA strains to be resistant to ciprofloxacin and cotrimoxazole, while $47.31 \%$ were resistant to amikacin. 
According to $\mathrm{S}$ Srinivasan et al, linezolid appears to be one of the few available antimicrobial agents with proven activity against MRSA including strains with reduced susceptibility to glycopeptides. ${ }^{12}$ They observed that $100 \%$ MRSA strains were within susceptibility range of linezolid by using both disc diffusion and MIC agar dilution method. This proves to be true for our study. As we have found that all MRSA strains were sensitive to linezolid, though we have used only Kirby-Bauer disc diffusion technique.

Clindamycin, was found to be another useful alternative in treating patients with MRSA. In a study by Shrinivasan $e t$., $l$ it was observed that all 50 isolates were sensitive to clindamycin. ${ }^{12}$ It is some what contrary to our findings, where we have observed more of sensitivity among all S.aureus isolates [(sensitive (45.20\%), moderately sensitive (16.66\%)] and of sensitivity among MRSA [sensitive (34.40\%), moderate sensitive (12.90\%)].

In a study conducted by Rajduraipandi et al., out 250 MRSA strains all (100\%) MRSA strains recorded sensitive to vancomycin, which was followed by $97.6 \%$ to linezolid ${ }^{75}$ Which were almost similar to our findings.

To conclude, MRSA prevalence in our hospital is $19.37 \%$, which is although not alarming, does not allow for complacency. Enforcing strict infection control practices will help us to tackle the situation. All MRSA isolates showed $100 \%$ sensitivity to vancomycin but nearly $75 \%$ isolate were having higher end of MIC which is a matter of concern for us. Though MRSA strains showed complete susceptibility to linezolid, it should be used very cautiously as a reservoir drug for serious MRSA infections.

\section{Conflicts of interest: None}

\section{References}

Kulkarni S, Khare A, Kour DC. Prevalence of methicillin resistant Staphylococcus aureus- A study in a tertiary care rural hospital. Ind $\mathbf{J}$ of basic and Applied Med Res. June 2014; 3(3): 414-421.

Thati V, Shivannavar CT, Gaddad SM. Vancomycin resistance among methicillin resistant Staphylococcus aureus isolates from intensive care units of tertiary care hospitals in Hyderabad. Indian J Med Res. Nov 2011; 134(5): 704-708

Rivera AM, Boucher HW. Current concepts in antimicrobial therapy against selected gram-positive organisms: Methicillin-resistant Staphylococcus aureus, penicillin-resistant Pneumococci, and vancomycinresistant Enterococci. Mayo Clin Proc. 2011; 86:1230-42.

Gram positive cocci Part I: Staphylococci and related Gram positive cocci In: Koneman's Color Atlas and Textbook of Diagnostic Microbiology. Koneman E W, Winn W C, Allen S D, Janda W M, Woods $G$ L editors. $6^{\text {th }}$ Edition Lippincott Williams and Wilkins, Baltimore 2006: 624-671.

Skov R, Smyth R, Larsen AR, Bolmstrôm A, Karlsson A, Mills K, et al. Phenotypic detection of methicillin resistance in Staphylococcus aureus by disk diffusion testing and Etest on MuellerHinton agar. J ClinMicrobiol 2006; 44 4395-9.

Clinical and Laboratory Standards Institute. Performance Standards for Antimicrobial Susceptibility Testing; Twenty-Fourth Informational Supplement. 11th ed. CLSI Document M100- S24.Wayne Pa: Clinical and 
Laboratory Standards Institute; Jan 2014.

Walters M, Lonsway D, Rasheed K, Albrecht, $\mathrm{V}$, McAllister, S, Limbago B, et al. Investigation and Control of Vancomycin-resistant Staphylococcus aureus: A Guide for Health Departments and Infection Control Personnel. Atlanta, GA 2015. Available at: http://www.cdc.gov/hai/pdfs/VRSAInvestigation-Guide-5_12_2015.pdf

Skov R, Smyth R, Larsen AR, Bolmstrôm A, Karlsson A, Mills K, et al. Phenotypic detection of methicillin resistance in Staphylococcus aureus by disk diffusion testing and Etest on MuellerHinton agar. J Clin Microbiol 2006; 44: 4395-9.

Anand KB, Agrawal P. Comparison of cefoxitin disc diffusion test, oxacillin screen agar and PCR for mecAgene for detection of MRSA. Indian J Med Microbiol 2009; 27(1): 27-9

Indian Network for Surveillance of Antimicrobial Resistance (INSAR) group, India. Methicillin resistant Staphylococcus aureus (MRSA) in India: Prevalence \& susceptibility pattern. Ind J Med Res February 2013: 363-69

Sharma P, Vishwanath G. Study of vancomycin susceptibility in methicillin resistant Staphylococcus aureus isolated from clinical samples. Annals of tropical medicine and Public health 2012; 5(3):178-180

Srinivasan S, Sheela D, Shashikala, Mathew R, Bazroy J. Risk factors and associated problems in the management of infections with methicillin resistant Staphylococcus aureus. Ind J of Med Microbiol 2006; 24(3): 182-5.

Sarma J B, Ahmed G U. Characterization of methicillin resistant $S$. aureus strains and risk factors for acquisition in a teaching hospital in northeast India.Ind $\mathrm{J}$ of Med Microbiol 2010; 28(2): 127129

Rajaduraipandi K, Mani KR, Panneerselvam K, Mani M, Bhaskar M, Manikandan P. Prevalence and antimicrobial susceptibility pattern of methicillin resistant Staphylococcus aureus: a multicentre study. Ind $\mathbf{J}$ of $\mathrm{Med}$ Microbiol 2006; 24 (1):34-38.

\section{How to cite this article:}

Prachi Arvind Anchawale. 2020. Prevalence of Methicillin Resistant Staphylococcus aureus (MRSA) and Multidrug Resistance among them in a Tertiary Care Rural Hospital. Int.J.Curr.Microbiol.App.Sci. 9(11): 778-784. doi: https://doi.org/10.20546/ijcmas.2020.911.094 